\title{
Characteristics of older adults that predict performance in building robotic kits
}

\author{
Eva L. Parkhurst MS ${ }^{a, *}$, Fernando Montalvo $\mathrm{BS}^{\mathrm{a}}$, Stella Sung $\mathrm{DMA}^{\mathrm{b}}$, Mar Navarro \\ $\mathrm{PhD}^{\mathrm{c}}$, Janan A. Smither $\mathrm{PhD}^{\mathrm{a}}$

\begin{abstract}
${ }^{a}$ Department of Psychology, University of Central Florida, Florida, USA; ${ }^{b}$ Center for Research and Education in Arts, Technology, and Entertainment (CREATE), University of Central Florida, Florida, USA; ' Department of Psychology, Ashford University, California, USA;
\end{abstract} \\ *Corresponding author: eva.parkhurst@ucf.edu
}

\begin{abstract}
Background: Robotic kits are a popular, modern pastime primarily marketed and designed for younger generations. Kits designed for use by older adults have the potential to promote cognitive, physical, and social engagement.

Objective: This study sought to determine characteristics and abilities of older adults that predicted efficiency, perceived usability, and engagement in building a market-available kit. Methods: Fifty-nine adults, 25 males, and 34 females, ages 55 to $87(M=70.80, S D=$ 8.44) completed a series of assessments for spatial visualization, visual abilities, working memory, and dexterity before assembling a robot kit.

Results: Backwards regression was utilized to create significant prediction models for five outcome variables: time on task, perceived system usability, task engagement, distress (i.e. negative affect), and worry (i.e. self-focused attention).

Conclusion: Results indicated that younger individuals and those with higher spatial visualization ability completed the build faster and perceived the kit to be more usable. Dexterity in the non-dominant hand also predicted faster build times. The findings of this study can be used to inform gerontechnological designs of assembly kits. Future studies can examine if the important factors identified here are predictive of performance in building more complex robotic kits.
\end{abstract}

Keywords: Robotic kits, leisure, product design

\section{INTRODUCTION}

The global population is in the midst of an unprecedented surge in individuals classified as older adults, defined as those aged 60 and older. From 1980 to 2017 the number of adults in this age category doubled to 962 million across the globe (UN, 2017). By 2050 that number is expected to double again, resulting in over 2 billion older individuals (UN, 2017).

This new reality has led to a concurrent growth in the field of gerontechnology which focuses on adapting and developing technologies aimed at the older populations. Even in the absence of chronic illnesses, aging is accompanied by physiological and cognitive changes that must be accounted for in designing for older adults (Simone \& Haas, 2009).

The current study focuses on build-at-home robotic kits, a modern pastime popular with children and young adults which has not been so readily accepted by older adults. Market-available kits often have design characteristics, such as small pieces and tools, which may prove frustrating to the older generations. The current project is one in a series intended to produce robot kits designed for use by older adults. Such kits can provide a new recreational activity for older adults that introduces and engages them with a new technology that promotes cognitive, physi$\mathrm{cal}$ and social engagement. The relative benefits of each is discussed below.

\section{Cognitive engagement}

Complexity of robot kits can vary widely due to factors such as the number of pieces, how the pieces are to be connected, and programming requirements. Regardless of these differences, the user must always reason through instructions while simultaneously building the robot, all the while recognizing and correcting any missteps.

\section{Cognitive function}

Research exploring the effect of mental exercise on continued cognitive function has elicited mixed results. Hughes, Sun, Change, \& Ganguli (2018), identified a positive relationship between total cognitive activities and intellectual functioning. Conversely, Salthouse (2006) compared activity levels and performance on cognitive tasks for participants aged 20 to 91 and found no evidence for the "use it or lose it" hypothesis that participation in cognitively challenging activities 
throughout the lifetime resulted in sustained cognitive functioning. Nevertheless, no evidence exists that engaging in cognitive activities does any harm to older adults, and the possibility of potential benefits is reason enough to continue such activities into old age (Salthouse, 2006). Additionally, Simone \& Haas (2009) identified that participation in leisure activities can influence the overall health and happiness of older adults.

\section{Physical engagement}

Building robotic kits rely heavily on the use of the upper limbs. Pieces and tools must be manipulated into place and grip strength is often required to connect pieces. While declines in motor function are present even in healthy aging individuals, evidence supports that continued use of the upper limbs and hands can help preserve higher levels of functionality.

\section{Hand-arm Function}

Decline in upper limb sensorimotor functions, such as gripping and manipulating objects, manifest even in healthy aging (Hackel, Wolfe, Bang, \& Canfield, 1992). Difficulty with precision, reduced hand/finger strength, and decrease in speed of movements are also common in older individuals (Bowden \& McNulty, 2013). These decrements in hand-muscle abilities are associated with a decreased ability to complete activities of daily living independently and thus can lead to a loss in quality of life (Gulde \& Hermsdörfer, 2017).

While the effect of mental exercise in prolonging cognitive function is unclear, the ability of even moderate physical exercise to prolong physical performance is well established across academic and medical domains. Targeted exercises have been shown to improve hand and finger strength in healthy individuals (e.g. Olafsdottir, Zatsiorsky \& Latash, 2008), as well as in those suffering from arthritis (e.g. Bergstra, Murgia, Te Velde, \& Caljouw, 2014). For those with arthritis, improvements in strength have been found to transfer to improvement in daily functioning without exacerbating arthritic pain (Bergstra, et al., 2014). Thus, even for individuals with hand dexterity and strength declines, activities that focus on these muscle groups may provide physical benefits.

Moreover, interventions that provide cognitive and motor components may be the most effective at helping individuals maintain their current level of function while simultaneously protecting against functional losses (Levin, Netz, \& Ziv, 2017). Robotic kits provide concurrent motor and mental challenges, as one must reason through instructions of the build while utilizing hand and arm movements to produce the requisite actions.

\section{Social engagement}

Contemporaneous with physical and cognitive stimulation, the building of robotic kits can also foster social engagement for older individuals. Loneliness and social isolation are growing concerns within the older adult populations. Builds can foster social connections when completed with peers and with younger individuals, such as grandchildren.

\section{Loneliness and social isolation}

Loneliness and social isolation can have deleterious effects on functioning and are both associated with an increased risk of mortality. Loneliness is a subjective emotional state where one feels disconnected or isolated from others. Social isolation can be objectively measured by quantifying the quality and amount of social contacts. In studying the objective measure of social isolation and the subjective feeling of loneliness, researchers have found that both are comparable to other risk factors, such as disease and smoking, in predicting mortality (Holt-Lunstad, et al., 2015).

Increased social contact can be a factor in curbing cognitive impairments, even in individuals with dementia. Bennett, Schneider, Tang, Arnold, and Wilson (2006) conducted a longitudinal study of elderly individuals with varying dementia diagnoses in which they collected periodic measures of cognitive function and social network size (measured as the number of visitors received per month) and, postmortem, analyzed the brain tissue of participants. Results indicated that participants with smaller social networks suffered the greatest decrements in cognitive function, regardless of specific diagnosis (Bennett et al., 2006).

Similarly, Biddle, et al. (2019) found that adults with the highest social engagement levels had a substantially reduced risk of developing Alzheimer's disease when compared to their less active counterparts. Studies confirming the positive effect of social networks on cognitive function have also noted that such relationships enhance the overall social engagement of older individuals. Furthermore, the interactions promote cognitive and physical activities which are also associated with higher independence and better quality of life (Simone \& Haas, 2009). Building robotic kits can provide an avenue for fostering social connections with peers and with younger generations.

\section{Intergenerational play}

Intergenerational play, especially play with grandchildren, has benefits for all parties. Grandchildren experience the comfort and security of a guardian while grandparents enjoy benefits that arise from teaching and nurturing (Agate, Agate, Liechty, \& Cochran, 2018). Intergenerational play taps into the benefits of both social networks and 
leisure activities, thereby providing older adults opportunities to be socially active and engaged.

Robotic kits are already targeted at the younger generations, with some kits recommended for children as young as three years old. Thus for many grandparents, building robots may already be of interest to their grandchildren. Expanding designs to encompass the skills and abilities of older individuals, introduces another avenue for intergenerational play.

\section{Current study}

The current study was intended to identify characteristics and abilities of older individuals that predicted performance, perceived usability, and engagement in building a market-available robotic kit. Participants were assessed for contrast sensitivity, spatial visualization, working memory, personality, and dexterity. These characteristics may be important predictive factors that can inform the design of kits to be built by older adults.

\section{Methods \\ Participants}

Participants were 59 community-dwelling older adults, 25 males and 34 females, ages 55 to 87 ( $M$ $=70.80, \mathrm{SD}=8.44)$. Participants were recruited from the general Orlando area through the Learning Institute for Elders (LIFE) at UCF, the Senior Tars (STARS) at Rollins, and community centers. To qualify for the study, participants had to score a minimum of 26 on the Montreal Cognitive Assessment (MoCA; Nasreddine et al., 2005).

\section{Materials}

A short demographics questionnaire was used to record information about age, ethnicity, highest educational level attained, and current or previous profession.

Utilizing a STEREO OPTEC 2000, visual acuity, color vision, and contrast sensitivity were assessed using the Snellen chart (Snellen, 1862), Ishihara test (Ishihara, 1972), and the Functional Acuity Contrast Test (FACT; Ginsburg, 1996), respectively. In the FACT test, the individual is shown circles containing gradient lines and must identify if the lines are oriented to the right, left, or vertically. The FACT uses five different sized gratings of varying spatial frequencies and nine contrast levels. The last grating orientation correctly identified by the participant is plotted on the contrast sensitivity curve.

The five-factor model of personality was measured using the Mini-International Personality Item Pool (Mini-IPIP; Donnellan, Oswald, Baird, \& Lucas, 2006). The Mini-IPIP uses 20 questions to measure levels of extraversion, agreeableness, conscientiousness, neuroticism, and intellect/im- agination (i.e. openness).

Spatial visualization is a class of spatial ability that refers to the ability to mentally rotate 2- and 3 -dimensional objects. Spatial visualization was assessed with the 30 questions Revised Purdue Spatial Visualizations Tests: Visualization of Rotations (Yoon, 2011). The test shows an exemplar three-dimensional object rotated along one or more axes. The participant is then shown a target object and asked to indicate which of five answer options represents the target if it was rotated along the same axes as the exemplar.

Working memory is the ability to temporarily retain and process mental information. Working memory capacity was assessed with an n-back task (Kirchner, 1958), specifically a computerized digit-span two-back task. Participants were presented with a list of numbers, one at a time, and were instructed to indicate when a number matched the target number from two steps prior. Participants completed three rounds, each consisting of 30 numbers presented. Accuracy was averaged across the rounds.

Dexterity of the hands and fingers was measured with the Perdue Pegboard, Model 32030. The board consists of four cups at the top containing small metal pins, washers, and collars. Below the cups are two parallel columns of 25 holes into which the pins can be inserted. Washers and collars can then be placed over a pin. The four tests administered with the Pegboard are a right-handed test, a left-handed test, a test using both hands, and an assembly test. In the first three, participants are given 30 seconds to place as many pins into the holes starting from the hole closest to the cups. The participant first completes the task with the dominant hand, then the non-dominant hand, and lastly with both hands simultaneously. The fourth task is a test of fine finger dexterity and requires participants to use both hands to form patterns of assemblies consisting of a pin, a washer, a collar, and another washer. The assembly task is scored as the total amount of pieces placed in one minute.

The System Usability Scale (SUS; Brooke, 1986) was used to assess the perceived usability of the robotic kit. The SUS consists of 10 items (e.g. I thought building this robot was easy to do) rated from "Strongly agree" to "Strongly disagree". The items were tailored to this experiment such that "robot" was substituted for "system". SUS scores are converted to percentile rankings and total ratings above 68 are considered above average usability for a system.

The Short Stress State Questionnaire (SSSQ; Helton, 2004) is a 24-item scale that measures 


\section{Older adults and robotic kits}

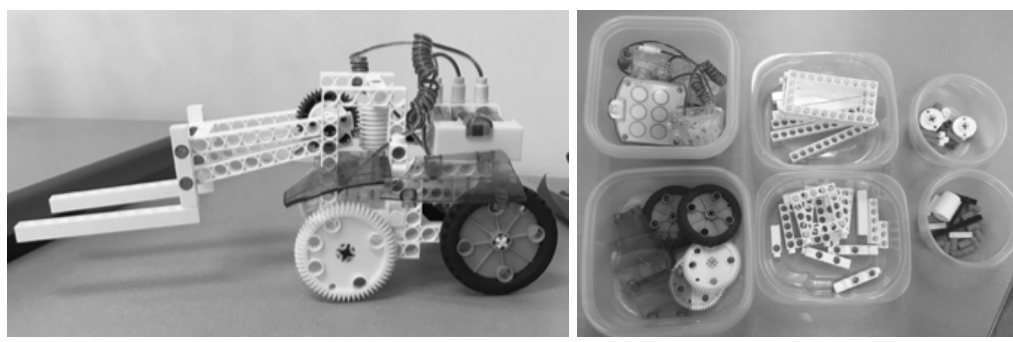

Figure 1. Completed build (left) and initial set-up of pieces (right).

three assessments were administered in random order. Subsequently, the participant completed the Pre-SSSQ survey.

After completion of all pre-surveys and assessments, participants were seated at a table with the instructions and pieces

pre and post-self-appraisals of task engagement, distress, and worry. The three scales represent aspects of stress states that are important to human performance: (1) task engagement refers to motivation, interest and focus on task, (2) distress is a measure of negative affect, and (3) worry is primarily a cognitive measure of self-focused attention and cognitive interference. Participants use a scale from 1 (Not at all) to 5 (Extremely) to rate how well a word describes them (e.g. Dissatisfied, Alert) and how well a statement describes their thoughts (e.g. I am committed to attaining my performance goals). The same items are presented before and after the task and final scores are derived by subtracting pre-scores from postscores for each subscale.

A qualitative, open-ended questionnaire was used to derive feedback about the overall build experience and impressions about the kit components including instructions and pieces.

The robot kit utilized in this study was the forklift build for the Thames \& Kosmos' Remote-Control Machines DLX (Figure 1). The authors conducted numerous build sessions with a variety of kits. The present kit was found to contain larger pieces, while not overly large, and could be connected without the use of a tool. Additionally, the selected build was estimated to require less than an hour to assemble. The instructions for the forklift build were provided in a bound, color booklet. Steps for the build were numbered by the manufacturer.

\section{Procedures}

Participants were asked to complete the demographics survey, the Mini-IPIP, and the spatial visualization test online prior to the in-lab session. A small portion of participants requested to complete the surveys in person and was given the opportunity to do so after completing the robot kit build.

In the lab session, participants were first screened with the MOCA and any participant who was unable to score a minimum of 26 was dismissed from the study. Those who completed the MOCA then completed the vision assessments, the n-back, and the Perdue Pegboard Test. The for the Remote-Control Machines DLX forklift build. Participants were given only the instructions and pieces required for the subject build. The pieces were divided into plastic containers with the same groupings for all participants (see Figure 1). Researchers drew attention to the illustration of the final build and highlighted the differences between two small types of connectors of the same color (see Figure 1). The participant was then instructed to work on the build alone but to ask the researcher if there were any questions or concerns.

Start and end times of each build were recorded. Additionally, every 15 minutes the researcher noted the participant's progress by recording the current step number. If the participant asked for help or seemed to be having difficulties, the researcher would assist as needed and log the step number and assistance details on a log sheet. Whenever possible participants were encouraged to work through any difficulties on their own. If a participant was still building after 90 minutes, the researcher would take a more active role in helping complete the build, while still allowing the participant to lead the process.

Once the forklift was complete, participants were given the remote control for the robot and given free rein to utilize the robot as desired. Participants chose to drive it around, use the forklift to transport objects, and some took videos or pictures.

Once the participants were done using the robot, they completed the Post-SSSQ and the SUS, in randomized order. Lastly, the researcher orally administered the qualitative questionnaire, after which the participant was debriefed and allowed time to ask questions about the study.

\section{Results AND DISCUSSION}

Backward linear regressions were conducted using SPSS to determine the best prediction model for each of the outcome variables - time on task, perceived system usability, task engagement, distress, and worry. The regression steps were done manually by excluding the variable with the lowest beta $(\beta)$ from the subsequent analysis until the model contained only significant predictors. The initially included predictors for all regressions are outlined in Table 1. 
Table 1. Predictors included in initial regression analysis.

\begin{tabular}{lc}
\hline Predictor & Measurement \\
\hline 1. Age & \\
\hline 2. Gender & \\
\hline 3. Contrast Sensitivity & Contrast sensitivity function \\
\hline 4. Spatial visualization & Spatial Visualization Test \\
\hline 5. Working memory & n-back \\
\hline 6. Dominant hand dexterity & Perdue Pegboard \\
7. Non-dominant hand & \\
dexterity & \\
8. Fine finger dexterity & \\
\hline 9. Agreeableness & \\
10. Conscientiousness & \\
11. Neuroticism & \\
12. Intellect/Imagination & \\
13. Extraversion & \\
\hline
\end{tabular}

Race/ethnicity and educational level were excluded due to a lack of variability among the sample. Contrast sensitivity was chosen as the best predictor of visual ability as it is considered a stronger indicator of real-world vision function than visual acuity (Norton, Corliss, \& Bailey, 2002). Contrast sensitivity requires individuals to distinguish images from a range of background contrasts as opposed to visual acuity, which tests vision under ideal lighting and contrast conditions (i.e. well lit black text on a white background).

Four female participants were excluded from analysis; three chose to end the study before completing the kit (one was uninterested in the activity and two stated that the kit was too difficult) and one did not complete the spatial visualization test. The age distribution was not significantly different for the remaining fifty-five participants $(M=70.20, S D=8.29 ; 21$ males and 34 females).

Table 2. Final regression model for time on task.

\begin{tabular}{lccc}
\hline Variable & $\mathbf{b}$ (SE) & $\mathbf{9 5} \% \mathbf{C l}$ & $\boldsymbol{\beta}$ \\
\hline Constant & 63.605 & {$[-.894$,} & \\
& $(32.128)$ & $128.105]$ & \\
\hline Spatial & -2.162 & {$[-2.886,-$} & -.546 \\
Visualization & $(.361)^{* * *}$ & $1.438]$ & \\
\hline Age & 1.033 & {$[.395$,} & .310 \\
& $(.318)^{* *}$ & $1.671]$ & \\
\hline Dexterity Non- & -3.375 & {$[-6.079,-$} & -.238 \\
Dominant & $(1.347)^{*}$ & $.671]$ & \\
Hand & & & \\
& & &
\end{tabular}

\begin{tabular}{lc}
\hline $\mathrm{R}^{2}$ & .592 \\
\hline $\mathrm{F}$ & $24.663^{* * *}$
\end{tabular}

Note. $\mathrm{N}=55 . \mathrm{Cl}=$ confidence interval; ${ }^{* * *} p<0.001 ;{ }^{* *} p<.01 ;{ }^{*} p<.05$.

\section{Time on task}

Time on task was measured as the total minutes from the start to the end of the build ( $M=$ $65.25, \mathrm{SD}=27.61)$. The results of the regression revealed spatial visualization, age, and dexterity in the non-dominant hand as significant predictors of time on task (Table 2). Spatial visualization was the strongest predictor $(\beta=-.546)$, with further stepwise regression analysis indicating that spatial visualization accounted for $39.9 \%$ of the variance in time on task.

Time on task was the main performance measure included in this study. Whereas participants were not instructed that they should complete the build as quickly as possible, the builds were conducted in quiet rooms with minimal distractions to encourage a focus on the task at hand.

High spatial visualization ability was the strongest predictor of faster completion times. The task required participants to manipulate pieces to match illustrated instructions. Those with the strongest ability to perform these manipulations mentally likely spent less time moving the actual pieces around as they matched components to the illustrations.

The younger age range of participants also predicted faster completion times. This study included individuals ranging from age 55 to 87 . The ages of 65 and 85 are traditionally considered milestones in aging with greater aging-related declines in cognitive and physical abilities accompanying each increasing age range. The significant contribution of age to performance is in line with this traditional model of aging.

Greater dexterity in the non-dominant hand was also predictive of less time on task. Building a robot kit requires constant use of both hands as one hand is often used to hold the current configuration while a piece is pressed into it. This particular kit also required the building of different sections of the robot and then bringing these sections together at a later step. The consistent reliance on both hands may explain why those with more dexterity in the non-dominant hand were able to complete the build more quickly.

\section{Perceived system usability}

The SUS is a widely-used and robust subjective measure of system usability. SUS scores above 68 are interpreted as average usability of the system. Scores above 68 indicate greater usability and scores below 68 less usabilities. Across participants, all measures of central tendency fell below the 68 cut-offs $(M=63.59$, Median = 65.00 , Mode $=65.00$ ), indicating that overall the participants perceived the kit used to be of less 
Table 3. Final regression model for worry (standardized).

\begin{tabular}{lccc}
\hline Variable & $\mathbf{b}(\mathbf{S E})$ & $\mathbf{9 5 \%} \mathbf{C l}$ & $\boldsymbol{\beta}$ \\
\hline Constant & -1.077 & {$[-1.730,-$} & \\
& $(.325)^{* *}$ & $4.24]$ & \\
\hline Working & 1.628 & {$[.885$,} & .547 \\
Memory & $(.370)^{* * *}$ & $2.370]$ & \\
\hline Neuroticism & -.030 & {$[-.049,-$} & -.358 \\
& $(.010)^{* *}$ & $.011]$ & \\
\hline Spatial & -.013 & {$[-.022,-$} & -.362 \\
Visualization & $(.005)^{* *}$ & $.004]$ & \\
\hline $\mathrm{R}^{2}$ & .372 \\
$\mathrm{~F}$ & & \\
\hline Note. $\mathrm{N}=55 . \mathrm{Cl}=$ confidence interval; & \\
\hline
\end{tabular}

than average usability. Scores ranged from 30 to 98 also indicating a large variance among individual participants.

Backwards regression identified spatial visualization and age as the statistically significant predictors of system usability ratings $(M=63.59, S D$ $=18.36) ; F(2,52)=16.798(p<.001), R^{2}=.392$, $\mathrm{R}_{\text {Adj }}{ }^{2}=.369, b_{\text {spatial }}=1.308(\mathrm{SEb}=.289), \beta_{\text {spatial }}=$ $.497, \mathrm{t}(54)=4.525, \mathrm{p}<.001 ; \mathrm{b}_{\mathrm{age}}=-.674\left(\mathrm{SE}_{\mathrm{b}}=\right.$ $.243), \beta_{\text {age }}=-.305, t(54)=-2.774, p<.01$. Spatial visualization ability was again the strongest predictor in the model, accounting for $30.3 \%$ of the variance in system usability ratings.

In line with time on task, higher kit usability ratings were associated with younger aged participants and those with higher spatial visualization scores. Therefore, these two predictors accounted for variance in objective measures of performance as well as the subjective perception of the usability of the kit overall. That is, participants that completed the build faster were also more likely to rate the system as more usable. Once again, the greatest predictor was the ability to mentally hold and manipulate objects.

\section{Distress}

To control for potential differences from the pre to post scores for the SSSQ scales, scores for task engagement, distress, and worry were standardized before analysis.

Dexterity in the dominant hand was the best predictor of variance in distress $(M=0.175, S D$ $=0.364)$ but the model was non-significant; $F(1$, $53)=2.924(p=.093), R^{2}=.052, R_{A d j}{ }^{2}=.034, b=$ $.039\left(\mathrm{SE}_{\mathrm{b}}=.023\right), \beta=.229, \mathrm{t}(54)=1.710, \mathrm{p}=.093$.

\section{Task engagement}

Regression analysis revealed contrast sensitivity and age as the significant predictors of task en- gagement, though the model accounted for only $13.9 \%$ of the variance $(M=0.062, S D=.163)$; $\mathrm{F}(2,52)=4.185(\mathrm{p}<.005), \mathrm{R} 2=.139, \mathrm{R}_{\mathrm{Adj}}{ }^{2}=.106$, $\mathrm{b}_{\text {contrast }}=.014(\mathrm{SEb}=.006), \beta_{\text {contrast }}=.347, \mathrm{t}(54)=$ $2.543, \mathrm{p}<.05 ;$ bage $=-.006\left(\mathrm{SE}_{\mathrm{b}}=.003\right), \beta_{\mathrm{age}}=$ $.291, \mathrm{t}(54)=2.138, \mathrm{p}<.05$.

Task engagement refers to focus and motivation in building the robotic kit. While not a strong predictor, age was significant in this model. Younger participants showed a greater increase in focus during the task.

As higher contrast sensitivity was the main predictor of task engagement, visual ability had some effect on the mental focus allotted to the task at hand. Sutter, Ladwig, Oehl \& Müsseler (2012) previously determined that older adults relied more on visual ability than proprioceptive feedback in the completion of motor tasks. The importance of visual ability in engagement may be reflected in this finding.

\section{Worry}

Three predictors were included in the final regression model which accounted for $37.2 \%$ of the variance in worry $(\mathrm{M}=-.57, \mathrm{SD}=.60$; Table 3$)$. Working memory $(\beta=.547)$ was the strongest predictor, while neuroticism ( $\beta=-.358$ ) and spatial visualization ( $\beta=-.362$ ) accounted for approximately equal portions of the remaining variance.

Worry is primarily a cognitive component of stress state that refers to cognitive intrusion from self-focused attention (Shelton, 2004). Higher working memory capacity was the largest predictor of increased worry scores. Thus, it seems that individuals with higher working memory scores were most apt to complete the build while also maintaining inward focus.

Lower spatial visualization also predicted a greater increase in worry scores, which may be a result of greater self-doubt with the reduced ability to mentally manipulate the pieces. As the entire build relied on object manipulation, this resulted in greater cognitive intrusion for these individuals.

Higher neuroticism scores predicted a stronger drop in worry scores. When examining pre- and post-robotic kit activity worry scores by the quartiles of neuroticism, it was evident that participants high in neuroticism had significantly higher pre-task worry scores $(M=20.70, S D=$ 4.72) than participants in the first $(M=14.60, S D$ $=4.42)$, second $(M=14.79, S D=5.11)$, and third quartiles $(M=14.73, S D=4.47)$ of neuroticism scores $\left(F(3,51)=4.37, p=.008, \eta_{p}^{2}=.20\right)$. This indicated that they were more worried about the task before they engaged in robotic kit building. However, after building the kit, scores on 
worry were lower for all groups, but were not significantly different between the neuroticism quartiles $\left(F(3,51)=0.98, \mathrm{p}=.41, \mathrm{n}_{\mathrm{p}}{ }^{2}=.05\right)$ indicating that the high neuroticism groups drop in worry score was highest. This is evident in the regression model which shows a more negative difference between pre- and post-scores for participants with higher neuroticism.

\section{Conclusions AND future ReSEARCH}

Build-at-home robotic kits are a popular, modern pastime primarily marketed and designed for younger generations. Kits designed for use by older adults have the potential to promote cognitive, physical, and social engagement. The regression model established for each outcome can aid in informing the selection and design of robotic kits for use by older adults.

Higher spatial visualization scores were significantly predictive of lower time on task, lower self-worry, and higher perceived system usability for the kit used in this study. Future research should focus on instructions and designs that may compensate for low spatial visualization skills. For instance, online instructions could allow for virtual rotation of pieces and configurations which may facilitate builds. Furthermore, creative designs may reduce the need for the rotation of pieces during the building process.

\section{References}

Agate, J. R., Agate, S. T., Liechty, T., \& Cochran, L. J. (2018). 'Roots and wings': An exploration of intergenerational play. Journal of Intergenerational Relationships, 16(4), 395-421.

Bennett, D., Schneider, J, Tang, Y., Arnold, S., \& Wilson, R. (2006). The effect of social networks on the relation between Alzheimer's disease pathology and level of cognitive function in old people: A longitudinal cohort study. The Lancet Neurology, 5(5), 406-412.

Bergstra, S. A., Murgia, A., Te Velde, A. F., \& Caljouw, S. R. (2014). A systematic review into the effectiveness of hand exercise therapy in the treatment of rheumatoid arthritis. Clinical Rheumatology, 33(11), 1539-1548.

Biddle, K. D., Uquillas, F. d'Oleire, Jacobs, H. I. L., Zide, B., Kirn, D. R., Rentz, D. M., Donovan, N. J. (2019). Social engagement and amyloid- $\beta$-related cognitive decline in cognitively normal older adults. The American Journal of Geriatric Psychiatry. https://doi-org.ezproxy.net.ucf.edu/10.1016/j. jagp.2019.05.005

Bowden, J. L., \& McNulty, P. A. (2013). The magnitude and rate of reduction in strength, dexterity and sensation in the human hand vary with ageing. Experimental Gerontology, 48(8), 756-765. https://doi. org/10.1016/j.exger.2013.03.011

Brooke, J. (1986). System usability scale (SUS): A quickand-dirty method of system evaluation user information. Reading, UK: Digital Equipment Co Ltd.
The significance of age in objective task performance and subjective appraisals of the kit usability highlights the importance of providing a range of complexity and difficulty levels in kit designs. Variability in kit design can then target a wider array of preferences and abilities.

The pieces of the current build were relatively large compared to other market-available kits. Furthermore, no tools were required. Nevertheless, the dexterity of the non-dominant hand had an influence on the performance of the build. Having older adults complete builds with a wider array of connector sizes and types can aid in establishing design guidelines for pieces that continue to provide physical engagement without exceeding capabilities.

Overall the current study identified important characteristics and abilities which influenced performance in building a robotic kit. Robotic kits represent a modern technology trend that can be introduced as a new pastime for older adults. Future studies can help determine if these factors are predictive of performance in building more complex kits. Additionally, the design of robotic kits that facilitate use by older adults should account for these factors, especially by identifying ways to bolster individuals with lower spatial visualization abilities.

Donnellan, M. B., Oswald, F. L., Baird, B. M., \& Lucas, R. E. (2006). The mini-IPIP scales: Tiny-yet-effective measures of the Big Five factors of personality. Psychological Assessment, 18(2), 192.

Ginsburg, A. P. (1996). Next generation contrast sensitivity testing. In Functional assessment of low vision (pp. 77-88). Mosby, St Louis, MO.

Gulde, P., \& Hermsdörfer, J. (2017). Both hands at work: The effect of aging on upper-limb kinematics in a multi-step activity of daily living. Experimental Brain Research, 235(5), 1337-1348.

Hackel, M. E., Wolfe, G. A., Bang, S. M., \& Canfield, J. S. (1992). Changes in hand function in the aging adult as determined by the Jebsen test of hand function. Physical Therapy, 72, 373-377.

Helton, W. S. (2004). Validation of a short stress state questionnaire. Proceedings of the Human Factors and Ergonomics Society, 48, 1238-1242.

Holt-Lunstad, J., Smith, T. B., Baker, M., Harris, T., \& Stephenson, D. (2015). Loneliness and social isolation as risk factors for mortality: A meta-analytic review. Perspectives on Psychological Science, 10(2), 227-237.

Hughes, T. F., Sun, Z., Chang, C. C. H., \& Ganguli, M. (2018). Change in engagement in cognitive activity and risk for mild cognitive impairment in a cohort of older adults. Alzheimer Disease \& Associated Disorders, 32(2), 137-144.

Ishihara, S. (1972). The Seventh Mental Measurements Yearbook (Vol. II). Buros, O. K. (Ed.). Lincoln, NE: University of Nebraska Press. 


\section{Older adults and robotic kits}

Kirchner, W. K. (1958). Age differences in short-term retention of rapidly changing information. Journal of Experimental Psychology, 55(4), 352-358.

Levin, O., Netz, Y., \& Ziv, G. (2017). The beneficial effects of different types of exercise interventions on motor and cognitive functions in older age: a systematic review. European Review of Aging and Physical Activity, 14(1), 20.

Nasreddine, Z. S., Phillips, N. A., Bedirian, V., Charbonneau, S., Whitehead, V., Collin, I., Cummings, J. L., \& Chertkow, (H. 2005) The Montreal Cognitive Assessment, MoCA: A brief screening tool for mild cognitive impairment. Journal of the American Geriatrics Society, 53(4), 695-699.

Norton, T. T., Corliss, D. A., \& Bailey, J. E. (2002). The psychophysical measurement of visual function (Vol. 362). Woburn: Butterworth-Heinemann.

Olafsdottir, H. B., Zatsiorsky, V. M., \& Latash, M. L. (2008). The effects of strength training on finger strength and hand dexterity in healthy elderly individuals. Journal of Applied Physiology, 105(4), 1166-1178.
Salthouse, T. A. (2006). Mental exercise and mental aging: Evaluating the validity of the "use it or lose it" hypothesis. Perspectives on Psychological Science, 1(1), 68-87.

Simone, P. M., \& Haas, A. L. (2009). Cognition and leisure time activities of older adults. LLI Review, 4, 22-28.

Snellen, H. (1862). Optotypi ad Visum Determinandum (Letterproeven tot Bepaling der Gezichtsscherpte; Probebuchstaben zur Bestimmung der Sehschaerfe). Utrecht, The Netherlands: Weyers.

Sutter, C., Ladwig, S., Oehl, M., \& Müsseler, J. (2012). Age effects on controlling tools with sensorimotor transformations. Frontiers in Psychology, 3(573), 1-8.

United Nations. (2017). World Population Ageing 2017 Highlights. Retrieved from: https://www.un.org/ en/development/desa/population/publications/pdf/ ageing/WPA2017_Highlights.pdf

Yoon, S. Y. (2011). Revised Purdue spatial visualization test: Visualization of rotations (Revised PSVT: R). Texas A\&M University, College Station, TX. 